\title{
Letter
}

\section{Eligibility for PrEP among MSM attending GUM clinics in the UK}

Ada R Miltz (MSc) $)^{1 *}$, Valentina Cambiano $(\mathrm{PhD})^{1}$, Fiona C Lampe $(\mathrm{PhD})^{1}$, Janey Sewell (BNurs $)^{1}$, Andrew Speakman $(\mathrm{PhD})^{1}$, Andrew N Phillips $(\mathrm{PhD})^{1}$, Daniel R Ivens (MBBS) ${ }^{2}$, David Asboe $(\mathrm{MBBS})^{3}$, Simon Collins ${ }^{4}$, Micheal Brady $(\mathrm{BMBS})^{5}$, Nneka C Nwokolo (MBBS) ${ }^{6}$ Alison J Rodger (MD) ${ }^{1}$; for the AURAH Study Group.

*Corresponding author, email: Ada.Miltz.11@ucl.ac.uk, telephone number: +44 2077940500 ext 38822

\section{Author affiliations}

${ }^{1}$ HIV Clinical Epidemiology and Biostatistics Group, Research Department of Infection and

Population Health, University College London, London, UK

${ }^{2}$ Royal Free Hospital, London, UK

${ }^{3}$ John Hunter Clinic, London, UK

${ }^{4} \mathrm{HIV}$ i-Base, London, UK

${ }^{5}$ King's College Hospital, London, UK

656 Dean Street, London, UK 
The National Health Service in England is currently discussing whether to fund a pre-exposure prophylaxis (PrEP) programme. The number of eligible individuals expected to come forward is a key consideration. The BHIVA/BASHH position statement supports access to PrEP for men who have sex with men (MSM) if they have a confirmed HIV-negative status, report condomless anal sex (CLS) with a man in the past three months, and report CLS is likely to occur again in the next three months ${ }^{1}$. We aimed to investigate what proportion of MSM attending genitourinary medicine (GUM) services could be eligible for PrEP in England, using data from the AURAH (Attitudes to and Understanding of Risk of Acquisition of HIV) study ${ }^{2}$, which recruited in English GUM services (2013-2014). A total of 4380 patients were approached over the study period and 2630 completed a questionnaire (response rate was $60 \%$ ). PrEP eligibility was assessed among 1484 gay, bisexual or other MSM. Of these men, 1227 had an HIV-test on the day of study recruitment, and 1220 had an HIV-negative test result (four positive test results and three missing). Of the HIV-negative men, 670 reported at least one episode of CLS in the past three months. Of the 550 HIV-negative men who did not report recent CLS, 106 reported bacterial STI diagnosis (past year), 38 reported post-exposure prophylaxis use (past year), and 79 reported chemsex associated drug use (methamphetamine, mephedrone or GHB/GBL) in the past three months; 178 reported at least one of these events. Of the 1484 MSM under assessment, 45.1\% (670/1484) had a confirmed HIV-negative status and reported recent CLS, and may represent the proportion of MSM GUM service attendees eligible for PrEP in England. An additional 12.0\% (178/1484) of these men may be eligible for PrEP, if other potential criteria are taken into consideration.

\section{AURAH study group}

Ada R. Miltz, Janey Sewell, Alison J. Rodger, Andrew Speakman, Fiona C Lampe, Andrew N. Phillips, Lorraine Sherr, Richard J. Gilson, David Asboe, Nneka C. Nwokolo, Amanda Clarke, Mark M. Gompels, Sris Allan, Simon Collins, Christopher Scott, Sara Day, Martin Fisher, Jane Anderson, Rebecca O'Connell, Monica Lascar, Vanessa Apea, Maneh Farazmand, Susan Mann, Jyoti Dhar, Daniel R. Ivens, Tariq Sadiq, Stephen Taylor, Michael Brady, Alan Tang, Rageshri Dhairyawan, Graham J. Hart, Anne M. Johnson, Alec Miners, and Jonathan Elford.

\section{References:}

1. McCormack S, Fidler S, Waters L, et al. BHIVA-BASHH Position Statement on PrEP in UK. Second update May 2016. United Kingdom: British HIV Association (BHIVA) and British Association for Sexual Health and HIV (BASHH), 2016. 
2. Sewell J, Speakman A, Phillips AN, et al. A Cross-Sectional Study on Attitudes to and Understanding of Risk of Acquisition of HIV: Design, Methods and Participant Characteristics. JMIR Res Protoc 2016;5(2):e58. 